\title{
The Estimation of Heart Weight from Routine Chest X-ray Films
}

\author{
Koichi Nagasawa, M.D., Eiichi Kmura, M.D., \\ and Chikio HAYashi, D.Sc.
}

\section{SUMmaRY}

A formula to estimate heart weight was derived using a theory of quantification which employed the measurements of cardiac silhouette taken from routine postero-anterior projection chest X-ray film. The films obtained in 73 necropsied cases of essential hypertension, renal hypertension, mitral and aortic valvular diseases, and 62 necropsied cases with other diseases were used for derivation of the formula. The measurements of the horizontal distances from the midsternal line to the intersecting point of the lower margin of each rib along the left cardiac border, and of the greatest distance along the right cardiac border were employed; body height, age and sex were also included.

The heart weight estimated by this formula coincided with the heart weight measured at necropsy with a high correlation ccefficient of 0.81 . The correlation rate in 34 external samples was also significantly high, being 0.72 . These results indicate the practical usefulness of this formula.

\section{Additional Indexing Words :}

Heart volume Cardiothoracic ratio Multivariate analysis

Theory of quantification

$7 \mathrm{HE}$ most widely used index for the estimation of cardiac size at present is the cardiothoracic ratio. ${ }^{11,2}$. When this ratio is larger than $50 \%$, the heart is regarded as enlarged. It is said, however, that a considerable number of false positive and false negative cases are present ${ }^{3 \prime}$ when comparing the cardiothoracic ratio with the actual heart weight measured at necropsy.

Therefore, it is essential that a more reliable but nonetheless simple method for estimation of heart weight is developed. The purpose of this paper is to describe a new method for the estiatmion of heart weight from roentgenogram,s using the theory of quantification, a kind of multivariate analysis, developed by Hayashi,"

From the First Department of Internal Medicine, Nippon Medical School, and Institute of Statistical Mathematics, Tokyo, Japan.

Address for reprint request to: Koichi Nagasawa, M.D., First Depastment of Inter nal Medicine, Nippon Medical School, 1-1-5 Sendagi, Bunkyo-ku, Tokyo 113, Japan.

Received for publication July 17, 1978. 


\section{Subjects AND Methods}

One hundred and thirty-five necropsied cases in our department, in which roentgenograms had been taken within 3 months prior to death, were used for the study. They consisted of 15 cases of essential hypertension, 19 cases of renal hypertension, 19 cases of mitral valvular disease, 20 cases of aortic valvular disease, and 62 cases of other diseases. Eighty-six cases were male and 49 were female, with the age ranging from 15 to 85 years. For the criteria of hypertension, systolic pressure of more than $160 \mathrm{mmHg}$ and/or diastolic pressure of more than $90 \mathrm{mmHg}$ were used. All cases of renal hypertension were accompanied by chronic renal insufficiency.

\section{Theory of quantification:}

The purpose of our study is to estimate the heart weight from the measurements of the cardiac silhouette. Since these measurements may not always be in linear relationship with the heart weight, effective results cannot always be obtained if the traditional method of linear regression analysis is employed.

The theory of quantification developed by Hayashi is applicable even to cases with non-linear relationships. According to this theory, the measurements (items) should be classified into several categories, and the numerical value to be given to each category is determined so as to meet the purpose most efficiently. The purpose in our study, for example, was to estimate the heart weight as accurately as possible. The sum of these values thus obtained will correspond to the estimated heart weight.

In more detail, we employ the transverse diameter of the cardiac silhouette as one of the parameters for the estimation of heart weight in this study. The values of this diameter obtained in a group of patients will be distributed over a considerable range. In the theory of quantification, this range should be divided into several sections, i.e. categories. Then the value of the transverse diameter in case $i$ belongs to one of the categories (category $k$ ) of an item $j$ (transverse diameter). Our purpose is to find $x_{j k}$, a numerical value given to category $k$ of item $j$, which makes the total of $x_{j k}$ as close as possible to the actual heart weight in case $i$.

This means that the estimated heart weight is expressed by the equation

$$
\alpha_{i}=\sum_{i} \sum_{k} \hat{o}_{i}(j k) x_{j k}
$$

where $\delta_{i}(j k)=1$, when case $i$ corresponds to category $k$ in item $j$; otherwise, $\delta_{i}(j k)=0$ and the correlation coefficient $\rho$ between the estimated and the actual heart weights is expressed as below ( $n$ is the number of cases),

$$
\begin{aligned}
& \rho=\frac{\frac{1}{n} \sum_{i}^{n}\left(A_{i}-\bar{A}\right)\left(\alpha_{i}-\bar{\alpha}\right)}{\sigma_{A} \sigma_{\alpha}} \\
& \sigma_{A}{ }^{2}=\frac{1}{n} \sum_{i}\left(A_{i}-\bar{A}\right)^{2} \quad \sigma_{a}{ }^{2}=\frac{1}{n} \sum_{i}\left(\alpha_{i}-\bar{\alpha}\right)^{2} \\
& \bar{A}=\frac{1}{n} \sum_{i} A_{i}, \quad \bar{\alpha}=\frac{1}{n} \sum_{i} \alpha_{i}
\end{aligned}
$$

Then the value $x$, which maximizes $\rho$, will be obtained by $\frac{\partial_{p}}{\partial x_{u v}}=0$, and 
the equation to be solved can be expressed as follows:

$$
\begin{aligned}
& \sum_{i}^{n} A_{i} \delta_{i}(u v)=x_{u v} n_{u v}+\Sigma^{\prime} \Sigma^{\prime} x_{j k} f(u v, j k) \\
& u=1,2, \ldots, R \quad v=1,2, \ldots, k_{u}
\end{aligned}
$$

where $R$ is the number of items, and $k_{u}$ is the number of categories in item $u$. The solution of this equation gives $x_{j k}$ which maximizes $\rho$.

2. The cardiac silhouette measurements employed in the study:

Routine postero-anterior projection chest roentgenograms taken at the time nearest to the death were used for the study, excluding those cases where pericardial effusion, massive pleural effusion or thoracic deformity were found.

In measuring the cardiac silhouette, the horizontal distances from the midsternal line to the intersecting points of the left cardiac border with lower margin of the ribs, $b_{1}, b_{2}, b_{3}, b_{4}$, and $b_{5}$, as seen in Fig. 1 , and the largest corresponding distance along the right cardiac border, $b_{R}$, were used. After examining the correlation between each measurement and the actual heart weight and the internal correlation between these measurements, the items indicated in Table I were selected as useful parameters for estimating the heart weight. Body height, age, and sex were added.

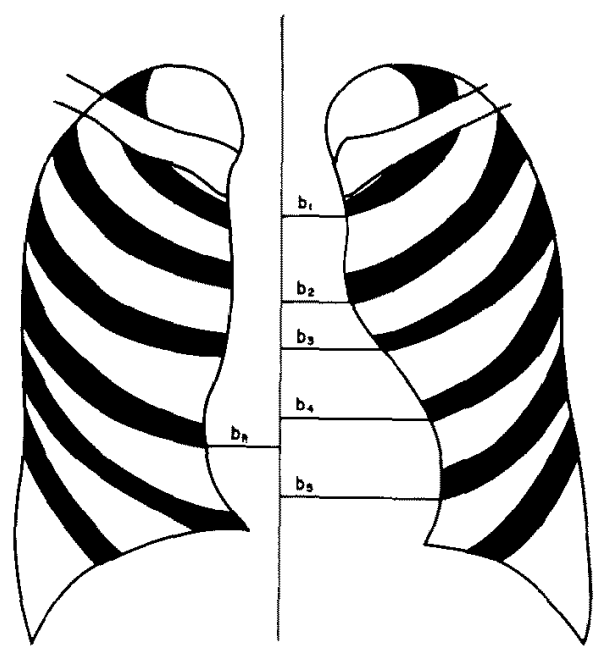

Fig. 1. The measurements of the cardiac silhouette in chest $\mathrm{X}$-ray films.

Table I. Parameters Selected for Estimation of the Heart Weight
(1) $b_{3}$
(6) $b_{R}$ /body height
(2) $b_{5}$
(7) Age
(3) $b_{R}$
(8) Sex
(4) $\Delta \mathrm{b}_{2}\left(=\mathrm{b}_{8}-\mathrm{b}_{2}\right)$
(9) Body height
(5) $b_{5} /$ body height 
Table II, Formula for the Estimation of the Heart Weight, Categorization of Measurements, and Quantitative Value of $x_{j k}$ to be substituted in the Formula

Quantitative value for substitution

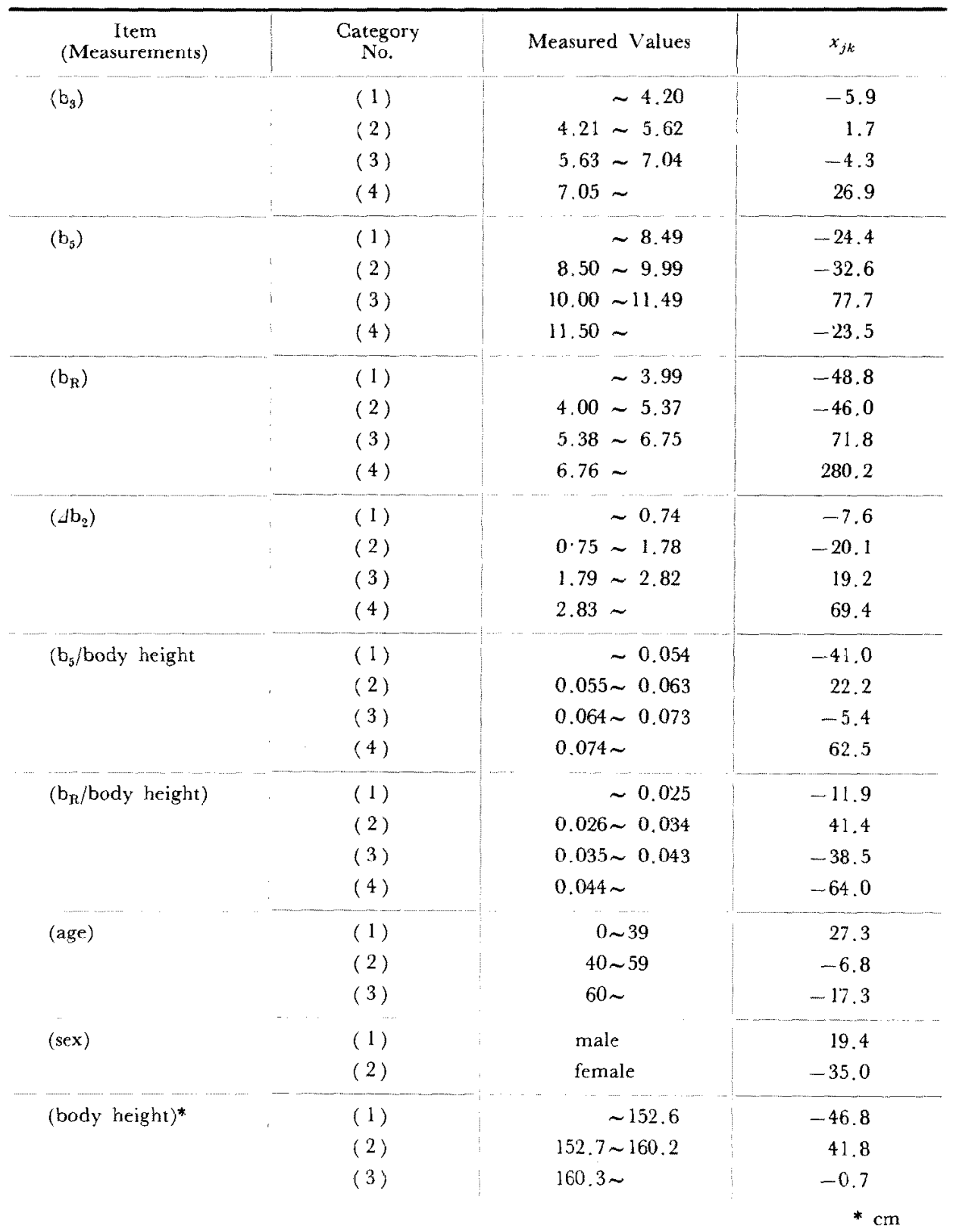

Estimated heart weight $=\left(b_{5}\right)+\left(b_{5}\right)+\left(b_{R}\right)+\left(J b_{2}\right)+\left(b_{5} /\right.$ body height $)+\left(b_{R} /\right.$ body height $)+($ age $)$ $+($ sex $)+($ body height $)+$ constant $(=420.0)$ 


\section{Results}

1. Correlation between the measurements of the cardiac silhouette and the heart weight:

Since no characteristic relationships were observed between each measurement and the actual weight in each individual disease group, we did not take the difference in diseases into consideration in estimating the heart weight.

\section{Categorization of the measurements:}

Each measurement of the cardiac silhouette was divided into 4 cateogries by employing the mean value " $m$ " and the standard deviation " $\sigma$ " of the frequency distribution function, as illustrated in Fig. 2. For body height, 3 categories were set with a standard deviation of $\pm 0.5 \sigma$ and for age, 3 categories-under 40,40 to 60 , and over 60 years-were established.

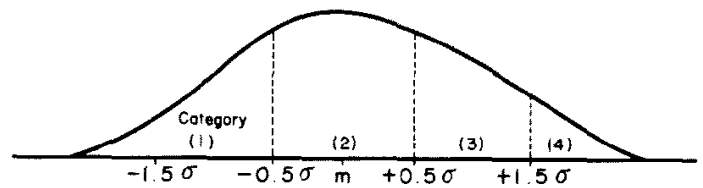

Fig. 2. Categorization of the measurements of the cardiac silhouette. " $\mathrm{m}$ ": mean value, " $\sigma ":$ standard deviation

3. Method of calculation:

Table II indicates the numerical values obtained by solving the formula (3) and given to each item-category. To obtain the estimated heart weight, if the value of $b_{3}$, for example, is between 4.21 and $5.62 \mathrm{~cm}$, the quantity 1.7 indicated in category (2) is substituted in the estimation formula shown on the bottom. The sum of the quantities substituted into the corresponding itemcategories gives the estimated heart weight.

4. Comparison of the estimated heart weight with the heart weight measured at necropsy:

As shown in Fig. 3, a highly significant correlation of 0.81 was obtained between the estimated heart weight and the actually measured heart weight at necropsy in 135 cases.

There were exceptions in only 30 cases, as seen in Table III, in which a difference more than $100 \mathrm{Gm}$ was observed between the 2 values. Suspected causes of disagreement are indicated in this table. Ascites was the most frequently observed cause of overestimation of the heart weight. On the other hand most of the cases where underestimation occurred had aortic valvular disease. In addition, in 19 out of a total of 20 cases with aortic valvular dis- 


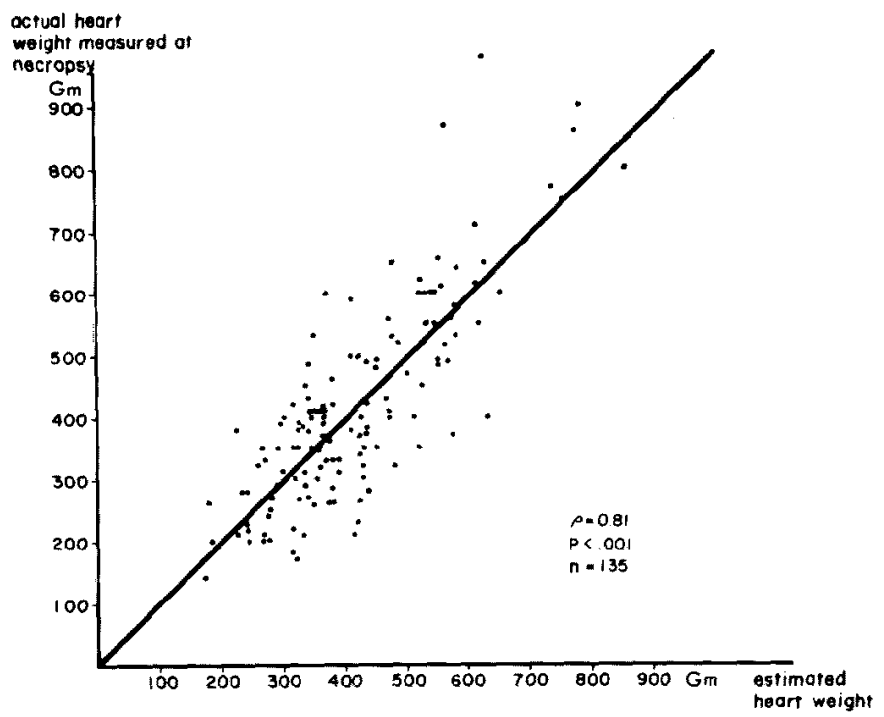

Fig. 3. Correlation between the estimated and the actual heart weights in 135 samples.

Table III. Cases showing a Difference of more than $100 \mathrm{Gm}$ between the Estimated and Actual Heart Weights

\begin{tabular}{l|c|c}
\hline & $\begin{array}{c}\text { Cases with over-estimated } \\
\text { heart weight }\end{array}$ & $\begin{array}{c}\text { Cases with under-estimated } \\
\text { heart weight }\end{array}$ \\
\hline Ascites & 10 & 1 \\
Pleural adhesion & 1 & 2 \\
Diaphragmatic adhesion & 1 & 0 \\
Enlarged heart & 1 & 1 \\
Aortic valvular disease & 0 & 0 \\
Unknown causes & 3 & 14 \\
\hline \multicolumn{1}{c|}{ Total } & 16 & 10 \\
\hline
\end{tabular}

ease the estimated heart weight was smaller than actually measured. Pleural and diaphragmatic adhesion and enlarged heart were also observed in a few cases.

5. Estimation of the heart weight in the external samples:

Fig. 4 shows the correlation for external samples between the estimated and the actual heart weights in 34 successive necropsied cases, including 13 cases with organic heart diseases, such as myocardial infarction, congenital, and acquired valvular heart diseases. As illustrated in this figure, a statistically significant correlation of 0.72 was observed. 


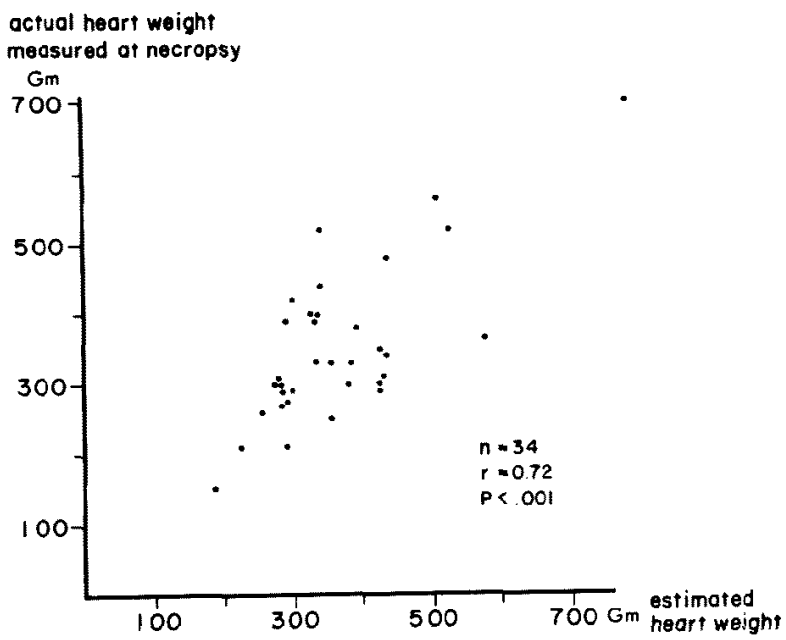

Fig. 4. Correlation between the estimated and the actual heart weights in 34 external samples.

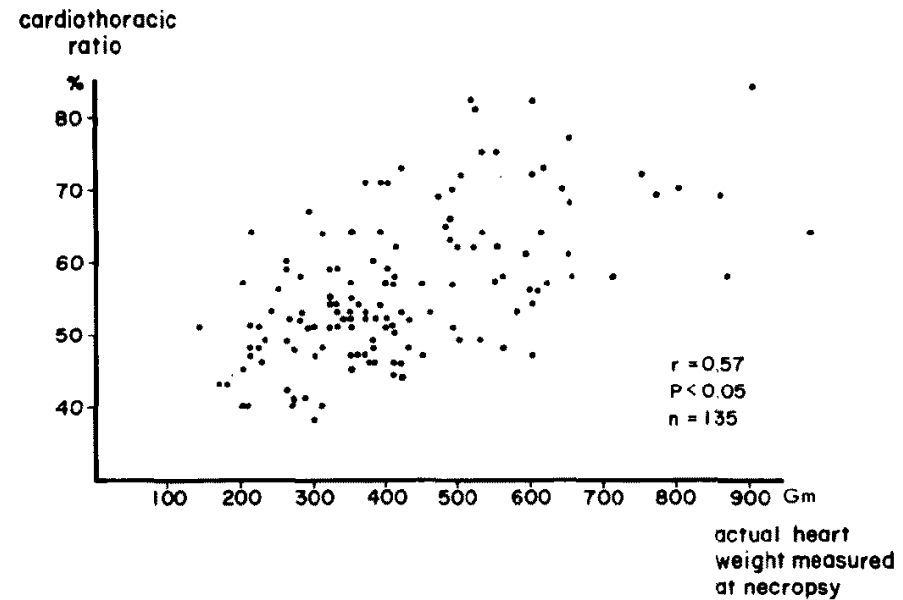

Fig. 5. Correlation between the cardiothoracic ratio and the actual heart weight in 135 samples.

6. Correlation between the cardiothoracic ratio and the actual heart weight:

Correlation between the cardiothoracic ratio and the actual heart weight was also examined in the 135 cases which were used for the derivation of the formula. The correlation coefficient was statistically significant, as seen in Fig. 5, but it was much smaller than that obtained using our estimation formula. 


\section{Discussion}

Estimation of the heart weight from the roentgenographic cardiac silhouette:

Although both the heart volume and the heart weight are regarded as indicating the size of heart, their clinical significance is not identical in the strict sense. In daily practice, however, we regard the larger heart as heavier and usually do not distinguish strictly between the heart size and the heart weight.

In addition to the cardiothoracic ratio, which is regarded as the simplest index for the heart size commonly employed, various methods for the evaluation of cardiac volume or cardiac surface area have been proposed using formulae or nomograms concerning the cardiac silhouette. ${ }^{51-8)}$ A method for the automatic formation of 3-dimensional real size image of the heart through the combination of real size figures obtained from several different projections ${ }^{91,101}$ has also been presented. We ${ }^{111}$ have also reported a method for the automatic estimation of heart volume using chest X-ray films taken in 6 projections. More precise estimation of the heart volume may be expected to be available through the use of the recently developed ACTAscanner, $\left.{ }^{12}\right)$

Since in the above-mentioned methods, however, some arbitrary factors are liable to be contained in the measurements or a large expensive equipment is often required, they cannot be considered sufficiently accurate and efficient at the present stage.

In our study, the heart weight was employed instead of the heart volume because the former is not only considered to be the more useful information as described above, but is regarded at necropsy as being a more important measurement than heart volume.

Only a few reports, however, have been published on the estimation of the weight of various organs by roentgenography. Whitley et al ${ }^{13)}$ estimated the spleen weight and $\operatorname{Ludin}^{14}$ the kidney weight with roentgenographic measurements.

Likewise, very few reports are available on estimating heart weight from electrocardiograms: Hugenholtz et $\mathrm{al}^{15}$ estimated the left ventricular weight from vectorcardiograms, using the linear quadratic discriminant function, and Kanie ${ }^{16}$ ) of our laboratory estimated the heart weight from scalar electrocardiographic measurements, using the theory of quantification employed in the present paper.

Problems in the measurement of the cardiac silhouette:

In order to measure the cardiac silhouette, it is desirable to use a roent- 
genogram taken under fixed conditions of respiration and cardiac cycle. In daily clinical practice, however, use of routine chest X-ray films is more convenient.

According to Kjellberg et al, ${ }^{17}$ no difference was observed in the cardiac silhouette between inspiration and expiration, nor was there any difference in cardiac volume between the systolic and the diastolic phases except in the cases of bradycardia. Moreover, in the automatic estimation of heart volume using AMDCOX (Automatic Measurement by Densitometry-Computer System of X-ray), which we have developed, ${ }^{11}$ we did not find any difference between the heart volume in systole and diastole. Cooley et $\mathrm{al}^{18}$ and Hilbish $^{19)}$ reported similar results.

Body constitution, body height, body weight, and chest diameter should be taken into consideration for the estimation of heart weight. Smith ${ }^{20}$ reported that a significant correlation existed between heart weight and body weight at necropsy, and Ludwig ${ }^{21}$ described the presence of a high correlation between the heart volume and body weight or chest diameter. In our study the correlation of heart weight with body height, body weight, and chest diameter were not significant, $r$ being $0.31,0.14,0.24$, respectively.

Because body height is, however, not so strongly influenced by the course of diseases, we included body height in addition to age and sex in our calculation.

\section{Evaluation of the results of estimation with our formula:}

The estimation formula derived by us is very simple and does not require complex calculation. Only the horizontal distances from the intersecting points of the cardiac border with the lower margin of each rib to the midsternal line are used.

Nevertheless, the heart weight estimated coincided quite closely with the actual heart weight measured at necropsy, showing a very high correlation coefficient of 0.81 . The high correlation coefficient of 0.72 was also obtained in 34 external samples, indicating the practical usefulness of this method.

These results were compared with the correlation coefficient between the cardiothoracic ratio and the actual heart weight measured at necropsy in these 135 cases. The correlation was inferior to the results obtained by our estimation formula, although it was statistically significant.

Discussion on the cases where the estimated heart weight differed markedly from the actual heart weight:

In 16 cases the estimated heart weight was larger by more than $100 \mathrm{Gm}$ 
than the weight measured at necropsy. More than half of these cases were found to have ascites, as indicated in Table III. It is not difficult to understand that the elevation of the diaphragm caused by the accumulation of ascites increases the values of $b_{5}$ and $b_{R}$, which have a close correlation with the heart weight, resulting in the overestimation.

The estimated heart weight was smaller than the actual weight by more than $100 \mathrm{Gm}$ in 14 cases, of which 10 cases had aortic valvular disease. This underestimation can be ascribed to the fact that the heart enlarges to the back in cases of aortic valvular diseases as the cardiomegaly advances. ${ }^{18}$ ) Therefore, it would be better to make an estimation formula specifically for aortic valvular disease.

In other cases with over- or underestimation, pleural and diaphragmatic adhesion or enlarged heart were observed; these also make the measurements of $b_{5}, b_{R}$, etc inaccurate. Since $b_{5}$, which is usually the largest distance in the left side of cardiac silhouette, might be not the largest in cases of low diaphragmatic position, it might be more appropriate to use $b_{1}$, the distance at the level of one rib lower, instead of $b_{5}$ in such cases.

Thus, the reasons for the disagreement of the weights were demonstrated in all but 3 cases, as indicated in Table III.

Needless to say, the separate use of the different estimation formula for each disease might increase accuracy, but would be very troublesome. Our results indicate that only one simple formula is sufficient for the estimation of the heart weight in daily practice.

\section{References}

1. Grocdel FM: Vereinfachte Ausmessung des Herz-orthodiagramms. Münch Med Wschr 65: 397,1918

2. Danzer CS: The cardiothoracic ratio. An index of cardiac enlargement. Am J Med Sci 157: 513,1919

3. Rosenfeld I, Goodrich C, Kassebaum G, Winston AL, Reader G: The electrocardiographic recognition of left ventricular hypertrophy. Am Heart J 63: 731, 1962

4. Hayashi C: Sample survey and theory of quantification. Bull Int Stat Inst 38: 505, 1961

5. Clagett $\mathrm{AH} \mathrm{Jr}$ : Cardiac roentgenology. The value of exact cardiac measurements. Am J Roentgenol 46: 794, 1941

6. Van Zwaluwenburg JG, Warren LF: The diagnostic value of the orthodiagram in heart disease. Arch Int Med 7: 137, 1911

7. Kjellberg SR: The roentgenologic determination of heart volume. Acta Med Scand 277 (suppl): 25, 1953

8. Hodges PC: Heart size from routine chest films. Radiology 47: 355, 1946

9. Lysholm E: Roentgenoskopischer Modellierungsapparat auch für Quersektion und Lokalisation. Acta Radiol 7: 189, 1926

10. Palmieri GG: Úber meine Methode der Plastischen Darstellung des Herzens am Lebenden (Radioplastik). Acta Radiol 10: 127, 1929 
11. Kimura E, Yamaguchi I, Kurokawa A, Hayashi G, Hayakawa H: Automatic estimation of heart volume by AMDCOX (Automatic Measurement by Densitometry-Computer System of X-ray). A preliminary report. Digest of the 10 th International Conference on Medical and Biological Engineering, Dresden, p 14, 1973

12. Ledley RS, Dichiro J, Luessenhop AJ, Twigg HL: Computerized transaxial X-ray tomography of the human body. Science 86: 207, 1974

13. Whitley JE, Maynard CD, Rhyne AL: A computer approach to the prediction of spleen weight from routine films. Radiology 86: 73, 1966

14. Ludin $\mathrm{H}$ : Radiologic estimation of kidney weight. Acta Radiol 6: 561, 1967

15. Hugenholtz PG, Ellison RC, Miettinen OS: Spatial voltages in the assessment of left ventricular hypertrophy (Frank system). J Electrocardiol 1: 77, 1968

16. Kanie T: Diagnosis of left ventricular hypertrophy and estimation of heart weight by electrocardiogram using the theory of quantification, a multivariate analysis. Jpn J Med 10: 156,1971

17. Kjellberg SR, Lönroth $\mathbf{H}$, Rudhe U: The effect of various factors on the roentgenological determination of the cardiac volume. Acta Radiol 35:413,1951

18. Cooley RN, Schreiber MH: Radiology of the Heart and Great Vessels. Williams \& Wilkins Co, Baltimore, 1967

19. Hilbish TF, Morgan RH: Cardiac mensuration by roentgenographic methods. Am J Med Sci 224: 586, 1952

20. Smith HL: The relation of the weight of the heart to the weight of the body and of the weight of the heart to age. Am Heart J 4: 79, 1928

21. Ludwig $\mathbf{H}$ : Roentgenologische Beurteilung der Herzgnösse. Fortschr Geb Rontgenstr Nuklearmed 59: 1, 1939 